\title{
A Class of Logarithmically Completely Monotonic Functions and Their Applications
}

\author{
Senlin Guo \\ Department of Mathematics, Zhongyuan University of Technology, Zhengzhou, Henan 450007, China \\ Correspondence should be addressed to Senlin Guo; sguo@hotmail.com
}

Received 7 April 2014; Accepted 10 May 2014; Published 13 July 2014

Academic Editor: Qiu-Ming Luo

Copyright (C) 2014 Senlin Guo. This is an open access article distributed under the Creative Commons Attribution License, which permits unrestricted use, distribution, and reproduction in any medium, provided the original work is properly cited.

We study the recent investigations on a class of functions which are logarithmically completely monotonic. Two open problems are also presented.

\section{Introduction}

Recall [1] that a positive function $f$ is said to be logarithmically completely monotonic (LCM) on an open interval $I$ if $f$ has derivatives of all orders on $I$ and for all $n \in \mathbb{N}:=$ $\{1,2,3, \ldots\}$,

$$
(-1)^{n}[\ln f(x)]^{(n)} \geq 0
$$

LCM functions are related to completely monotonic (CM) functions [2], strongly logarithmically completely monotonic (SLCM) functions [3], almost strongly completely monotonic (ASCM) functions [3], almost completely monotonic (ACM) functions [4], Laplace transforms, and Stieltjes transforms and have wide applications. It is evident that the set of SLCM functions is a nontrivial subset of the set of LCM functions, which is a nontrivial subset of the set of CM functions, and that the set of CM functions is a nontrivial subset of the set of ACM functions. It was established [3] that the set of SLCM functions is a nontrivial subset of the set of ASCM functions and that the set of SLCM functions on the interval $(0, \infty)$ is disjoint with the set of strongly completely monotonic (SCM) functions (see [5] for its definition) on the interval $(0, \infty)$.

It is well known that the classical Euler gamma function is defined for $x>0$ by

$$
\Gamma(z)=\int_{0}^{\infty} t^{x-1} e^{-t} \mathrm{~d} t
$$

The logarithmic derivative of $\Gamma(z)$, denoted by

$$
\psi(z)=\frac{\Gamma^{\prime}(z)}{\Gamma(z)},
$$

is called psi function, and $\psi^{(k)}$ for $k \in \mathbb{N}$ are called polygamma functions.

For $\alpha, \gamma \in \mathbb{R}$ and $\beta \geq 0$, define

$$
f_{\alpha, \beta, \gamma}(x):=\left[\frac{e^{x} \Gamma(x+\beta)}{x^{x+\beta-\alpha}}\right]^{\gamma}, \quad x \in(0, \infty),
$$

which is encountered in probability and statistics.

Since $f_{\alpha, \beta, \gamma}(x)(\gamma>0)$ is logarithmically completely monotonic if and only if $f_{\alpha, \beta, 1}(x)$ is logarithmically completely monotonic and $f_{\alpha, \beta, \gamma}(x)(\gamma<0)$ is logarithmically completely monotonic if and only if $f_{\alpha, \beta,-1}(x)$ is logarithmically completely monotonic, we only need to study the logarithmically complete monotonicity of the function

$$
f_{\alpha, \beta, \pm 1}(x)=\left[\frac{e^{x} \Gamma(x+\beta)}{x^{x+\beta-\alpha}}\right]^{ \pm 1}, \quad x \in(0, \infty) .
$$

In [6, Theorem 3.2], it was proved that the function $f_{1 / 2,0,1}(x)$ is decreasing and logarithmically convex from $(0, \infty)$ onto $(\sqrt{2 \pi}, \infty)$ and that the function $f_{1,0,1}(x)$ is increasing and logarithmically concave from $(0, \infty)$ onto $(1, \infty)$. 
In [7, Theorem 1], for showing

$$
\frac{b^{b-1}}{a^{a-1}} e^{a-b}<\frac{\Gamma(b)}{\Gamma(a)}<\frac{b^{b-1 / 2}}{a^{a-1 / 2}} e^{a-b}
$$

for

$$
b>a>1
$$

monotonic properties of the functions $\ln f_{\alpha, 0,1}(x)$ and $\ln f_{\alpha, 0,1}(x)$ on the interval $(1, \infty)$ were obtained.

In [8, Theorem 2], it was presented that the function $f_{\alpha, 0,1}(x)$ is decreasing on the interval $(c, \infty)$ for $c \geq 0$ if and only if

$$
\alpha \leq \frac{1}{2}
$$

and increasing on the interval $(c, \infty)$ if and only if

$$
\alpha \geq \begin{cases}c[\ln c-\psi(c)] & \text { if } c>0, \\ 1 & \text { if } c=0 .\end{cases}
$$

In [9], after proving the logarithmically completely monotonic property of the functions $f_{1 / 2,0,1}(x)$ and $f_{1,0,-1}(x)$, in virtue of Jensen's inequality for convex functions, the upper and lower bounds for the Gurland's ratio were established: for positive numbers $x$ and $y$, the inequality

$$
\frac{x^{x-1 / 2} y^{y-1 / 2}}{[(x+y) / 2]^{x+y-1}} \leq \frac{\Gamma(x) \Gamma(y)}{[\Gamma((x+y) / 2)]^{2}} \leq \frac{x^{x-1} y^{y-1}}{[(x+y) / 2]^{x+y-2}}
$$

holds true, where the middle term in (10) is called Gurland's ratio [10].

In [11] the authors proved the following result.

Theorem 1 (see [11]). If

$$
2 \alpha \leq 1 \leq \beta,
$$

then the function $f_{\alpha, \beta, 1}(x)$ is logarithmically completely monotonic on the interval $(0, \infty)$.

The necessary and sufficient conditions for the functions $f_{\alpha, 0,1}(x)$ and $f_{\alpha, 0,-1}(x)$ to be logarithmically completely monotonic on the interval $(0, \infty)$ were also given in [11].

Using monotonic properties of the functions $f_{1 / 2,0,1}(x)$ and $f_{1,0,-1}(x)$, the inequality (6) was extended (see [11, Remark 1]) from

$$
b>a>1
$$

to

$$
b>a>0 .
$$

In [12] the authors proved the following results.

Theorem 2 (see [12]). If $\beta>0$ and $\alpha \leq 0$, then the function $f_{\alpha, \beta, 1}(x)$ is logarithmically completely monotonic on the interval $(0, \infty)$.
Theorem 3 (see [12]). For $\beta>0$, a necessary condition for the function $f_{\alpha, \beta, 1}(x)$ to be logarithmically completely monotonic on the interval $(0, \infty)$ is that

$$
\alpha \leq \min \left\{\beta, \frac{1}{2}\right\}
$$

Theorem 4 (see [12]). For $\beta \geq 1$, a necessary and sufficient condition for the function $f_{\alpha, \beta, 1}(x)$ to be logarithmically completely monotonic on the interval $(0, \infty)$ is that

$$
\alpha \leq \frac{1}{2}
$$

As direct consequences of the above results, the following Kečkić-Vasić-type inequality is deduced.

Theorem 5 (see [12]). Let $x$ and $y$ be positive numbers with $x \neq y$.

(1) For $\beta \geq 1$, the following inequality

$$
I(x, y)>\left[\left(\frac{x}{y}\right)^{\alpha-\beta} \frac{\Gamma(x+\beta)}{\Gamma(y+\beta)}\right]^{1 /(x-y)}
$$

holds true if and only if $\alpha \leq 1 / 2$, where

$$
I(a, b)=\frac{1}{e}\left(\frac{b^{b}}{a^{a}}\right)^{1 /(b-a)} \quad(a>0, b>0, a \neq b)
$$

is the identric or exponential mean.

(2) For $\beta>0$, the inequality (16) holds true also if $\alpha \leq 0$.

In [13], the following result was established.

Theorem 6 (see [13]). (1) For $\beta \in[0,1 / 2)$, if

$$
\alpha \leq \beta-e^{-4}(1-\beta)^{2} \exp \left(\frac{2}{1-\beta}\right)
$$

then the function $f_{\alpha, \beta, 1}(x)$ is logarithmically completely monotonic on the interval $(0, \infty)$.

(2) For $\beta \in[1 / 2,1]$, if

$$
\alpha \leq \min \left\{3 \beta^{2}-3 \beta+1, \frac{1}{2}\right\}
$$

then the function $f_{\alpha, \beta, 1}(x)$ is logarithmically completely monotonic on the interval $(0, \infty)$.

From Theorem 6 we can directly obtain the following new result.

Corollary 7. (1) For $\beta \in[1 / 4,1 / 2]$, if

$$
\alpha \leq \beta-\frac{1}{4},
$$

then the function $f_{\alpha, \beta, 1}(x)$ is logarithmically completely monotonic on the interval $(0, \infty)$. 
(2) For $\beta \in(1 / 2,3 / 4]$, if

$$
\alpha \leq \beta-\frac{1}{3},
$$

then the function $f_{\alpha, \beta, 1}(x)$ is logarithmically completely monotonic on the interval $(0, \infty)$.

(3) For $\beta \in(3 / 4,1]$, if

$$
\alpha \leq \beta-\frac{1}{2},
$$

then the function $f_{\alpha, \beta, 1}(x)$ is logarithmically completely monotonic on the interval $(0, \infty)$.

A necessary and sufficient condition is obtained in [13] as follows.

Theorem 8 (see [13]). For

$$
\beta \in\{0\} \cup\left[\frac{1}{2}+\frac{\sqrt{3}}{6}, \infty\right),
$$

a necessary and sufficient condition for the function $f_{\alpha, \beta, 1}(x)$ to be logarithmically completely monotonic on the interval $(0, \infty)$ is that

$$
\alpha \leq \frac{1}{2} .
$$

Regarding the logarithmically complete monotonicity for the function $f_{\alpha, \beta,-1}(x)$ and their applications. In [14], the authors proved the following results.

Theorem 9 (see [14]). If the function $f_{\alpha, \beta,-1}(x)$ is logarithmically completely monotonic on the interval $(0, \infty)$, then either

$$
\beta>0, \quad \alpha \geq \max \left\{\beta, \frac{1}{2}\right\}
$$

or

$$
\beta=0, \quad \alpha \geq 1 .
$$

Theorem 10 (see [14]). For

$$
\beta \geq \frac{1}{2},
$$

the necessary and sufficient condition for the function $f_{\alpha, \beta,-1}(x)$ to be logarithmically completely monotonic on the interval $(0, \infty)$ is that

$$
\alpha \geq \beta .
$$

As first application, the following inequalities are derived by using logarithmically completely monotonic properties of the function $f_{\alpha, \beta, \pm 1}(x)$ on the interval $(0, \infty)$.

Theorem 11 (see [14]). (1) For $k \in \mathbb{N}$, double inequalities

$$
\begin{gathered}
\ln x-\frac{1}{x} \leq \psi(x) \leq \ln x-\frac{1}{2 x}, \\
\frac{(k-1) !}{x^{k}}+\frac{k !}{2 x^{k+1}} \leq(-1)^{k+1} \psi^{(k)}(x) \leq \frac{(k-1) !}{x^{k}}+\frac{k !}{x^{k+1}}
\end{gathered}
$$

hold true on the interval $(0, \infty)$.
(2) When $\beta>0$, inequalities

$$
\begin{gathered}
\psi(x+\beta) \leq \ln x+\frac{\beta}{x}, \\
(-1)^{k} \psi^{(k-1)}(x+\beta) \geq \frac{(k-2) !}{x^{k-1}}-\frac{\beta(k-1) !}{x^{k}}
\end{gathered}
$$

hold true on the interval $(0, \infty)$ for $k \geq 2$.

(3) When $\beta \geq 1 / 2$, inequalities

$$
\begin{gathered}
\psi(x+\beta) \geq \ln x, \\
(-1)^{k} \psi^{(k-1)}(x+\beta) \leq \frac{(k-2) !}{x^{k-1}}
\end{gathered}
$$

hold true on the interval $(0, \infty)$ for $k \geq 2$.

(4) When $\beta \geq 1$, inequalities

$$
\begin{gathered}
\psi(x+\beta) \leq \ln x+\frac{\beta-1 / 2}{x} \\
(-1)^{k} \psi^{(k-1)}(x+\beta) \geq \frac{(k-2) !}{x^{k-1}}-\frac{(\beta-1 / 2)(k-1) !}{x^{k}}
\end{gathered}
$$

hold true on the interval $(0, \infty)$ for $k \geq 2$.

As second application, the following inequalities are derived by using logarithmically convex properties of the function $f_{\alpha, \beta, \pm 1}(x)$ on $(0, \infty)$.

Theorem 12 (see [14]). Let $n \in \mathbb{N}$ and

$$
x_{k}>0 \quad(1 \leq k \leq n) .
$$

Suppose also that

$$
\sum_{k=1}^{n} p_{k}=1 \quad\left(p_{k} \geq 0\right)
$$

If either

$$
\beta>0, \quad \alpha \leq 0
$$

or

$$
\beta \geq 1, \quad \alpha \leq \frac{1}{2}
$$

then

$$
\frac{\prod_{k=1}^{n}\left[\Gamma\left(x_{k}+\beta\right)\right]^{p_{k}}}{\Gamma\left(\sum_{k=1}^{n} p_{k} x_{k}+\beta\right)} \geq \frac{\prod_{k=1}^{n} x_{k}^{p_{k}\left(x_{k}+\beta-\alpha\right)}}{\left(\sum_{k=1}^{n} p_{k} x_{k}\right)^{\sum_{k=1}^{n} p_{k} x_{k}+\beta-\alpha}} .
$$

If

$$
\alpha \geq \beta \geq \frac{1}{2},
$$

then the inequality (37) reverses.

As final application, the following inequality can be derived by using the decreasingly monotonic property of the function $f_{\alpha, \beta,-1}(x)$ on $(0, \infty)$. 
Theorem 13 (see [14]). If

$$
\alpha \geq \beta \geq \frac{1}{2}
$$

then

$$
I(x, y)<\left[\left(\frac{x}{y}\right)^{\alpha-\beta} \frac{\Gamma(x+\beta)}{\Gamma(y+\beta)}\right]^{1 /(x-y)}
$$

holds true for $x, y \in(0, \infty)$ with $x \neq y$, where $I(x, y)$, defined by (17), is the identric or exponential mean.

The following results were shown in [15].

Theorem 14 (see [15]). For

$$
\beta \geq 0
$$

a sufficient condition for the function $f_{\alpha, \beta,-1}(x)$ to be logarithmically completely monotonic on the interval $(0, \infty)$ is that

$$
\alpha \geq \max \left\{\frac{1}{2}, \beta, 3 \beta^{2}-3 \beta+1\right\} .
$$

Remark 15. From Theorems 9 and 14 we see that the necessary and sufficient condition for the function $f_{\alpha, 0,-1}(x)$ to be logarithmically completely monotonic on the interval $(0, \infty)$ is that

$$
\alpha \geq 1
$$

This result is Theorem 2 in [11]. Here we recovered it.

Theorem 16 (see [15]). Let

$$
\beta \in\left[\frac{1}{2}-\frac{\sqrt{3}}{6}, \frac{1}{2}\right] .
$$

Then the necessary and sufficient condition for the function $f_{\alpha, \beta,-1}(x)$ to be logarithmically completely monotonic on the interval $(0, \infty)$ is that

$$
\alpha \geq \frac{1}{2}
$$

The following results are applications of the above theorems.

Theorem 17 (see [15]). When

$$
\frac{1}{2}-\frac{\sqrt{3}}{6} \leq \beta \leq \frac{1}{2}
$$

the following inequalities

$$
\begin{gathered}
\psi(x+\beta) \geq \ln x-\frac{1 / 2-\beta}{x}, \\
(-1)^{k} \psi^{(k-1)}(x+\beta) \leq \frac{(k-2) !}{x^{k-1}}+\frac{(1 / 2-\beta)(k-1) !}{x^{k}} \\
(k \geq 2)
\end{gathered}
$$

hold true on the interval $(0, \infty)$.
Theorem 18 (see [15]). Let $n \in \mathbb{N}$ and

$$
x_{k}>0 \quad(1 \leq k \leq n) .
$$

Suppose also that

$$
\sum_{k=1}^{n} p_{k}=1 \quad\left(p_{k} \geq 0\right)
$$

If

$$
0 \leq \beta \leq \frac{1}{2},
$$

$$
\alpha \geq \max \left\{\frac{1}{2}, 3 \beta^{2}-3 \beta+1\right\}
$$

then

$$
\frac{\prod_{k=1}^{n}\left[\Gamma\left(x_{k}+\beta\right)\right]^{p_{k}}}{\Gamma\left(\sum_{k=1}^{n} p_{k} x_{k}+\beta\right)} \leq \frac{\prod_{k=1}^{n} x_{k}^{p_{k}\left(x_{k}+\beta-\alpha\right)}}{\left(\sum_{k=1}^{n} p_{k} x_{k}\right)^{\sum_{k=1}^{n} p_{k} x_{k}+\beta-\alpha}} .
$$

Theorem 19 (see [15]). If

$$
\begin{gathered}
0 \leq \beta \leq \frac{1}{2}, \\
\alpha \geq \max \left\{\frac{1}{2}, 3 \beta^{2}-3 \beta+1\right\},
\end{gathered}
$$

then

$$
\begin{gathered}
I(x, y)<\left[\left(\frac{x}{y}\right)^{\alpha-\beta} \frac{\Gamma(x+\beta)}{\Gamma(y+\beta)}\right]^{1 /(x-y)} \\
(x>0 ; y>0 ; x \neq y),
\end{gathered}
$$

where in (53) $I(x, y)$, defined by (17), is the identric or exponential mean.

\section{Open Problems}

2.1. Open Problem 1. From Theorem 8 we have already known, for

$$
\beta \in\{0\} \cup\left[\frac{1}{2}+\frac{\sqrt{3}}{6}, \infty\right),
$$

a necessary and sufficient condition for the function $f_{\alpha, \beta, 1}(x)$ to be logarithmically completely monotonic on the interval $(0, \infty)$.

For

$$
\beta \in\left(0, \frac{1}{2}+\frac{\sqrt{3}}{6}\right),
$$

what is a necessary and sufficient condition for the function $f_{\alpha, \beta, 1}(x)$ to be logarithmically completely monotonic on the interval $(0, \infty)$ ?

Already Known. Theorem 3 gave a necessary condition; Theorem 6 provided a sufficient condition. 
2.2. Open Problem 2. From Remark 15, Theorems 10 and 16 we have already known, for

$$
\beta \in\{0\} \cup\left[\frac{1}{2}-\frac{\sqrt{3}}{6}, \infty\right),
$$

a necessary and sufficient condition for the function $f_{\alpha, \beta,-1}(x)$ to be logarithmically completely monotonic on the interval $(0, \infty)$.

For

$$
\beta \in\left(0, \frac{1}{2}-\frac{\sqrt{3}}{6}\right),
$$

what is a necessary and sufficient condition for the function $f_{\alpha, \beta,-1}(x)$ to be logarithmically completely monotonic on the interval $(0, \infty)$ ?

Already Known. Theorem 9 gave a necessary condition; Theorem 14 provided a sufficient condition.

\section{Conflict of Interests}

The author declares that there is no conflict of interests regarding the publication of this paper.

\section{Acknowledgments}

The author would like to thank the editor and the reviewers for their valuable comments and suggestions to improve the quality of this paper. The present investigation was supported, in part, by the Natural Science Foundation of China under Grant no. 11326167.

\section{References}

[1] R. D. Atanassov and U. V. Tsoukrovski, "Some properties of a class of logarithmically completely monotonic functions," Comptes Rendus de l'Académie Bulgare des Sciences, vol. 41, no. 2, pp. 21-23, 1988.

[2] D. V. Widder, The Laplace Transform, Princeton University Press, Princeton, NJ, USA, 1941.

[3] S. Guo and H. M. Srivastava, "A certain function class related to the class of logarithmically completely monotonic functions," Mathematical and Computer Modelling, vol. 49, no. 9-10, pp. 2073-2079, 2009.

[4] H. M. Srivastava, S. Guo, and F. Qi, "Some properties of a class of functions related to completely monotonic functions," Computers \& Mathematics with Applications, vol. 64, no. 6, pp. 1649-1654, 2012.

[5] S. Y. Trimble, J. Wells, and F. T. Wright, "Superadditive functions and a statistical application," SIAM Journal on Mathematical Analysis, vol. 20, no. 5, pp. 1255-1259, 1989.

[6] G. D. Anderson, R. W. Barnard, K. C. Richards, M. K. Vamanamurthy, and M. Vuorinen, "Inequalities for zero-balanced hypergeometric functions," Transactions of the American Mathematical Society, vol. 347, no. 5, pp. 1713-1723, 1995.

[7] J. D. Kečkić and P. M. Vasić, "Some inequalities for the gamma function," Institut Mathématique, vol. 11, no. 25, pp. 107-114, 1971.
[8] H. Alzer, "On some inequalities for the gamma and psi functions," Mathematics of Computation, vol. 66, no. 217, pp. 373389, 1997.

[9] Y.-J. Wei, S.-L. Zhang, and C.-P. Chen, "Logarithmically completely monotonic functions and Gurland's ratio for the gamma functions," Advanced Studies in Contemporary Mathematics, vol. 15, no. 2, pp. 253-257, 2007.

[10] M. Merkle, "Gurland's ratio for the gamma function," Computers \& Mathematics with Applications, vol. 49, no. 2-3, pp. 389406, 2005.

[11] C.-P. Chen and F. Qi, "Logarithmically completely monotonic functions relating to the gamma function," Journal of Mathematical Analysis and Applications, vol. 321, no. 1, pp. 405-411, 2006.

[12] S. Guo, F. Qi, and H. M. Srivastava, "Supplements to a class of logarithmically completely monotonic functions associated with the gamma function," Applied Mathematics and Computation, vol. 197, no. 2, pp. 768-774, 2008.

[13] S. Guo and H. M. Srivastava, "A class of logarithmically completely monotonic functions," Applied Mathematics Letters, vol. 21, no. 11, pp. 1134-1141, 2008.

[14] S. Guo, F. Qi, and H. M. Srivastava, "A class of logarithmically completely monotonic functions related to the gamma function with applications," Integral Transforms and Special Functions, vol. 23, no. 8, pp. 557-566, 2012.

[15] S. Guo, "Logarithmically completely monotonic functions and applications," Applied Mathematics and Computation, vol. 221, pp. 169-176, 2013. 


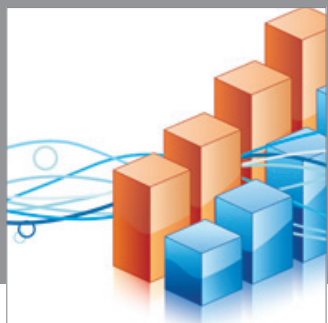

Advances in

Operations Research

mansans

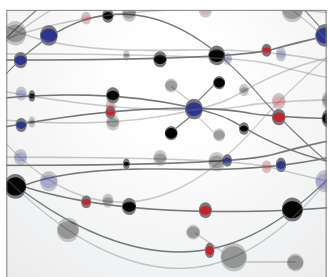

The Scientific World Journal
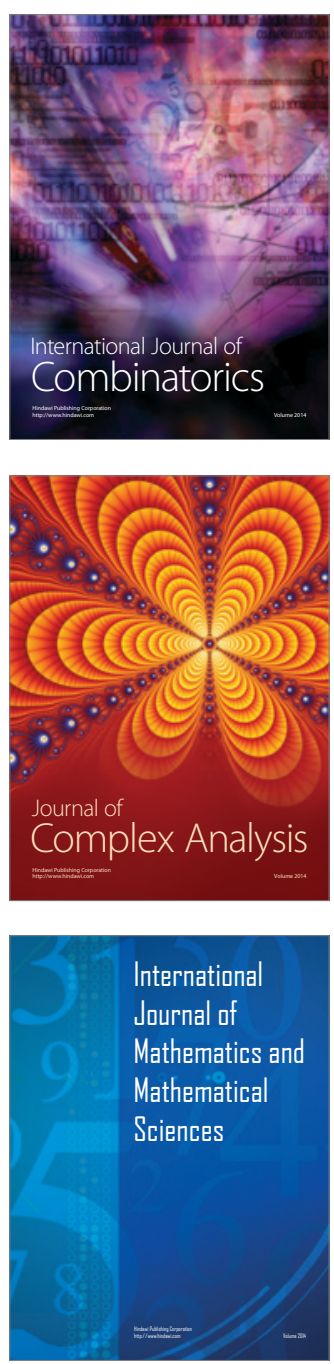
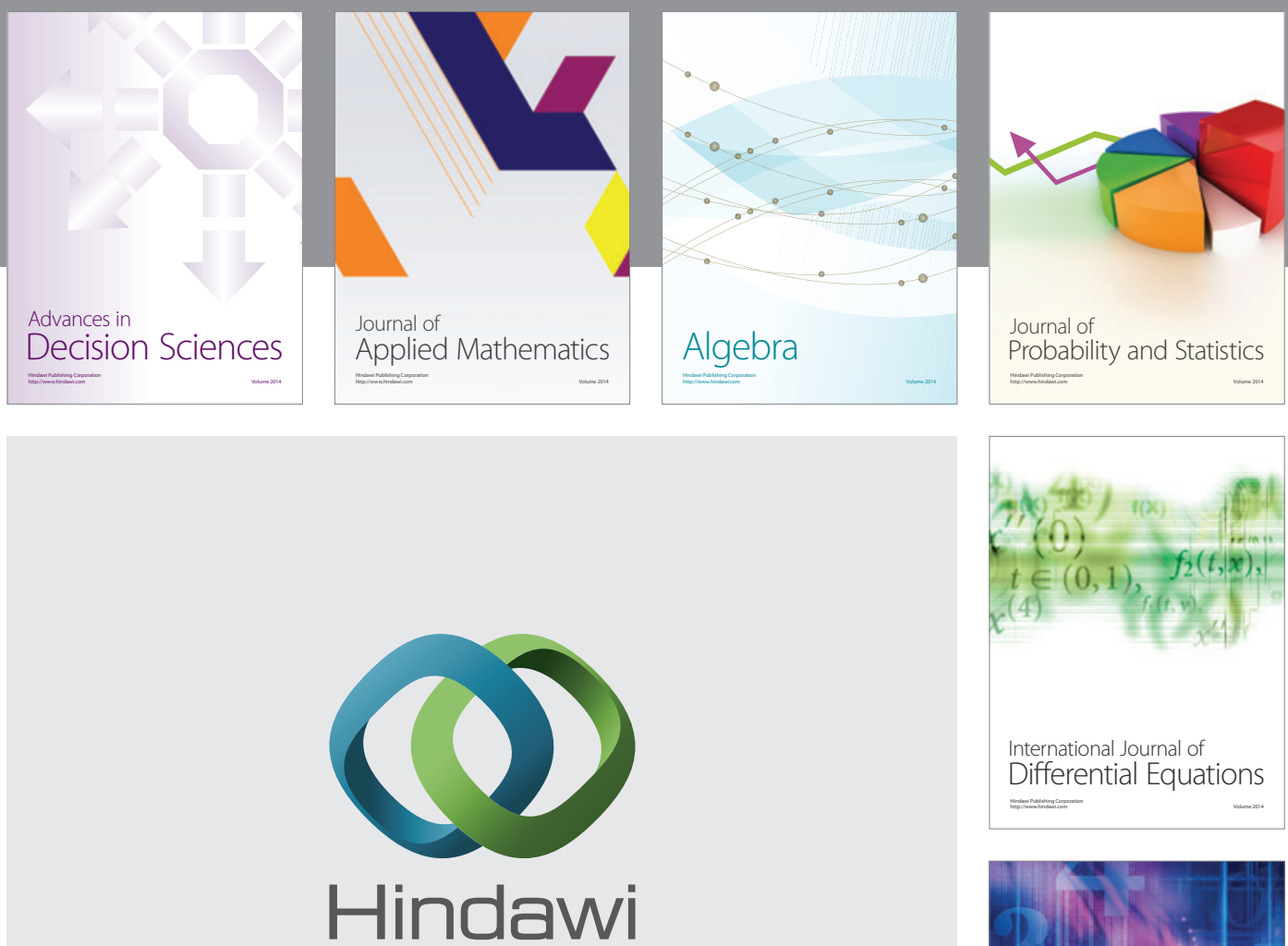

Submit your manuscripts at http://www.hindawi.com
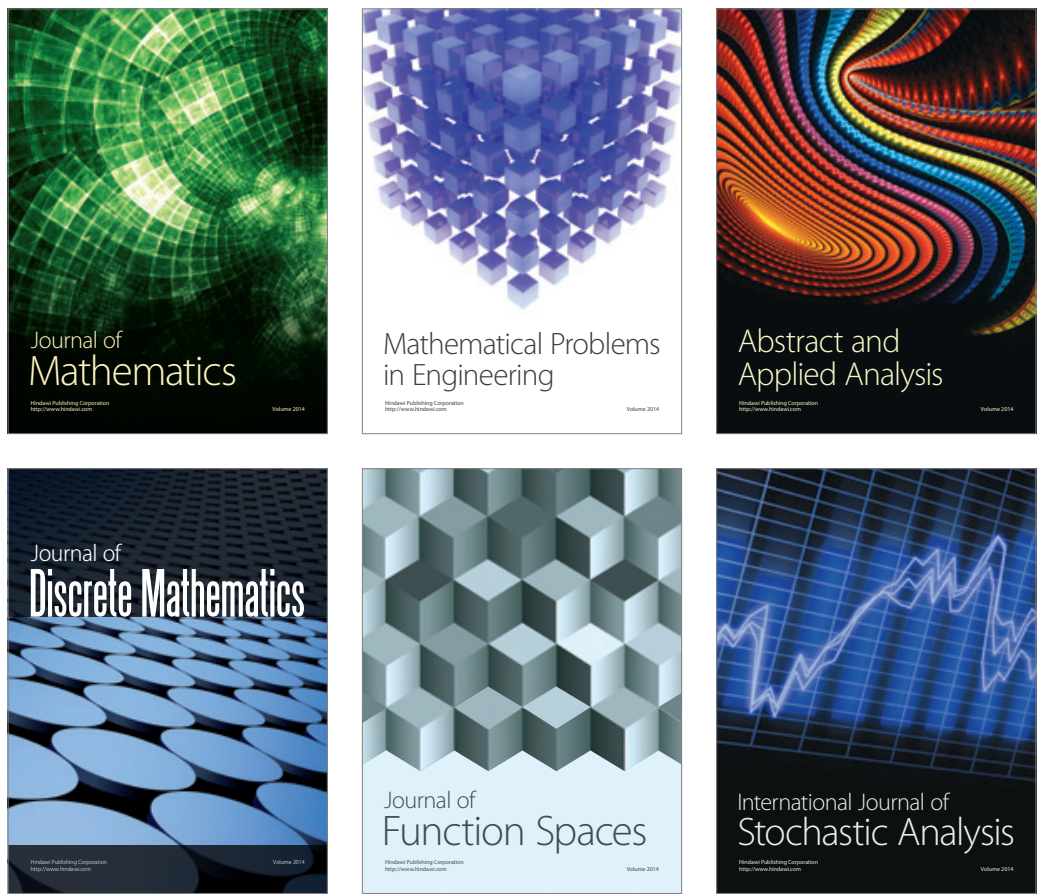

Journal of

Function Spaces

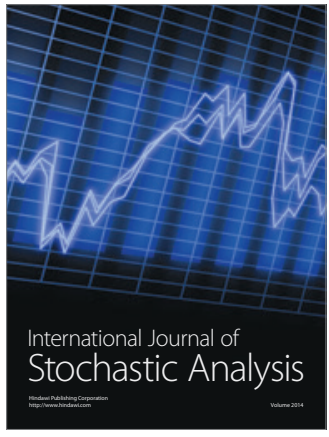

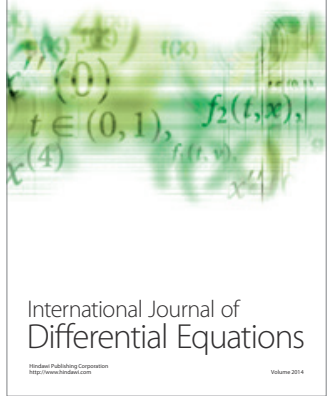
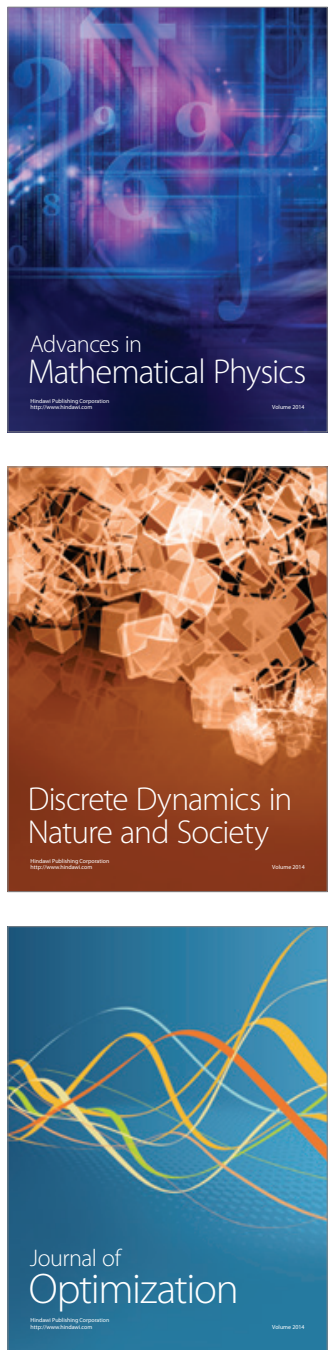